\title{
Time-Resolved Coherent Diffraction of Ultrafast Structural Dynamics in a Single Nanowire
}

\author{
Marcus C. Newton, ${ }^{1,2, *}$ Mayu Sao, ${ }^{3}$ Yuta Fujisawa, ${ }^{3}$ Rena Onitsuka, ${ }^{4,}{ }^{5}$ Tomoya Kawaguchi, ${ }^{6}$ \\ Kazuya Tokuda, ${ }^{6}$ Takahiro Sato, ${ }^{4}$ Tadashi Togashi, ${ }^{7}$ Makina Yabashi, ${ }^{4}$ Tetsuya Ishikawa, ${ }^{4}$ \\ Tetsu Ichitsubo, ${ }^{6}$ Eiichiro Matsubara, ${ }^{6}$ Yoshihito Tanaka, ${ }^{4,5}$ and Yoshinori Nishino ${ }^{3}$ \\ ${ }^{1}$ Research Institute for Electronic Science, Hokkaido University \\ ${ }^{2}$ Department of Physics \&S Astronomy, University of Southampton, UK \\ ${ }^{3}$ Research Institute for Electronic Science, Hokkaido University, Sapporo 001-0021, Japan \\ ${ }^{4}$ RIKEN SPring-8 Center, RIKEN, 1-1-1, Kouto, Sayo, Hyogo 679-5148, Japan \\ ${ }^{5}$ Department of Physics, School of Science and Technology, \\ Kwansei Gakuin University, Gakuen, Sanda, Hyogo 669-1337, Japan \\ ${ }^{6}$ Department of Materials Science and Engineering, Kyoto University, Kyoto 606-8501, Japan \\ ${ }^{7}$ Japan Synchrotron Radiation Research Institute (JASRI), \\ 1-1-1 Kouto, Sayo-cho, Sayo-gun, Hyogo 679-5198, Japan
}

(Dated: April 11, 2014)

\begin{abstract}
The continuing effort to utilise the unique properties present in a number of strongly correlated transition metal oxides for novel device applications has led to intense study of their transitional phase state behaviour. Here we report on time-resolved coherent X-ray diffraction measurements on a single vanadium dioxide nanocrystal undergoing a solid-solid phase transition, using the SACLA X-ray Free Electron Laser (XFEL) facility. We observe an ultra-fast transition from monoclinic to tetragonal crystal structure in a single vanadium dioxide nanocrystal. Our findings demonstrate that the structural change occurs in a number of distinct stages attributed to differing expansion modes of vanadium atom pairs.
\end{abstract}

16 Correlated electronic materials are those in which 17 the electronic interactions are not readily predicted 18 through a study of the individual constituents. These 19 materials often undergo a phase transition when sub20 ject to excitation, where quantities including either 21 electronic structure, crystal structure or magnetic or22 dering are significantly altered. This process is often 23 driven by a change in temperature but may also oc${ }_{24}$ cur due to electrical, mechanical, optical and magnetic 25 excitations.[1-4]

26 Simple models of correlated systems have proven ${ }_{27}$ difficult to solve due to the large number of inter28 actions that must be accounted for. As a result, 29 accurately simulating theoretical predictions is often 30 impractical and leaves little to guide experimental 31 studies. [5] There remains therefore the challenge to 32 develop a dynamical theory that is able to describe 33 spontaneous atomic rearrangement due to external ex34 citation. In addition, there are few studies dedicated 35 to time resolved measurements. This is largely due to 36 the limitations of the available tools for studying such 37 systems. For example, electron microscopes are able 38 to resolve atomic scale features of sufficiently thin ma39 terials but are in general limited to pico-second time 40 resolution. [6] The key to deciphering these phenom${ }_{41}$ ena lies in measuring the time-dependent changes in 42 atomic structure. Observation of the dynamical be43 haviour is permitted only when the time scale for mea44 surement is appropriately less than that of the phase ${ }_{45}$ change phenomena.

${ }_{46}$ Vanadium dioxide $\left(\mathrm{VO}_{2}\right)$ is a transition metal ox47 ide material that exhibits a solid-solid phase transi-
48 tion from tetragonal to monoclinic atomic ordering 49 at a critical temperature $T_{c}=67.9^{\circ} \mathrm{C}$ which is ac50 companied by a metal-to-insulator phase transition. 51 In the monoclinic (tetragonal) phase, $\mathrm{VO}_{2}$ belongs to ${ }_{52}$ the $P 2_{1} / c\left(P 4_{2} / m n m\right)$ space group respectively. The 53 structural change in $\mathrm{VO}_{2}$ can occur on the femto54 second timescale, as demonstrated here. There is 55 still however some debate on the nature of the transi56 tion due to conflicting results, with authors subscrib57 ing to either the Mott-Hubbard or Peierls dominated 58 interactions.[7-10] A change in conductivity, several 59 orders of magnitude in size, across $T_{c}$ has made $\mathrm{VO}_{2}$ 60 a long standing topic of research with the aim of de${ }_{61}$ veloping potential device applications where ultra-fast ${ }_{62}$ switching characteristics are desirable.[11-14]

63 The advent of $4^{\text {th }}$ generation X-ray Free-Electron ${ }_{64}$ Laser (XFEL) facilities, as a tool for materials analy$65 \mathrm{sis}$, is proven to provide unprecedented insight into 66 nanometre scale structure. [15-18] These studies ${ }_{67}$ demonstrate the potential that high brilliance light 68 sources have for advancing our understanding of a 69 wide range of phenomena. Coherent X-ray diffraction 70 (CXD) imaging is a powerful lens-less imaging tool for 71 probing materials with nanometer resolution.[19] Con72 ventional CXD is performed by illuminating a sample 73 with a spatially coherent X-ray source so that the co74 herence area exceeds the dimensions of the crystal; a 75 condition that is always satisfied for an XFEL source, 76 when the X-ray beam size is larger than the sam77 ple. In the Bragg reflection geometry, scattered light 78 from the volume of the crystal interferes to produce 79 a coherent diffraction pattern in the far-field.[20-22] 


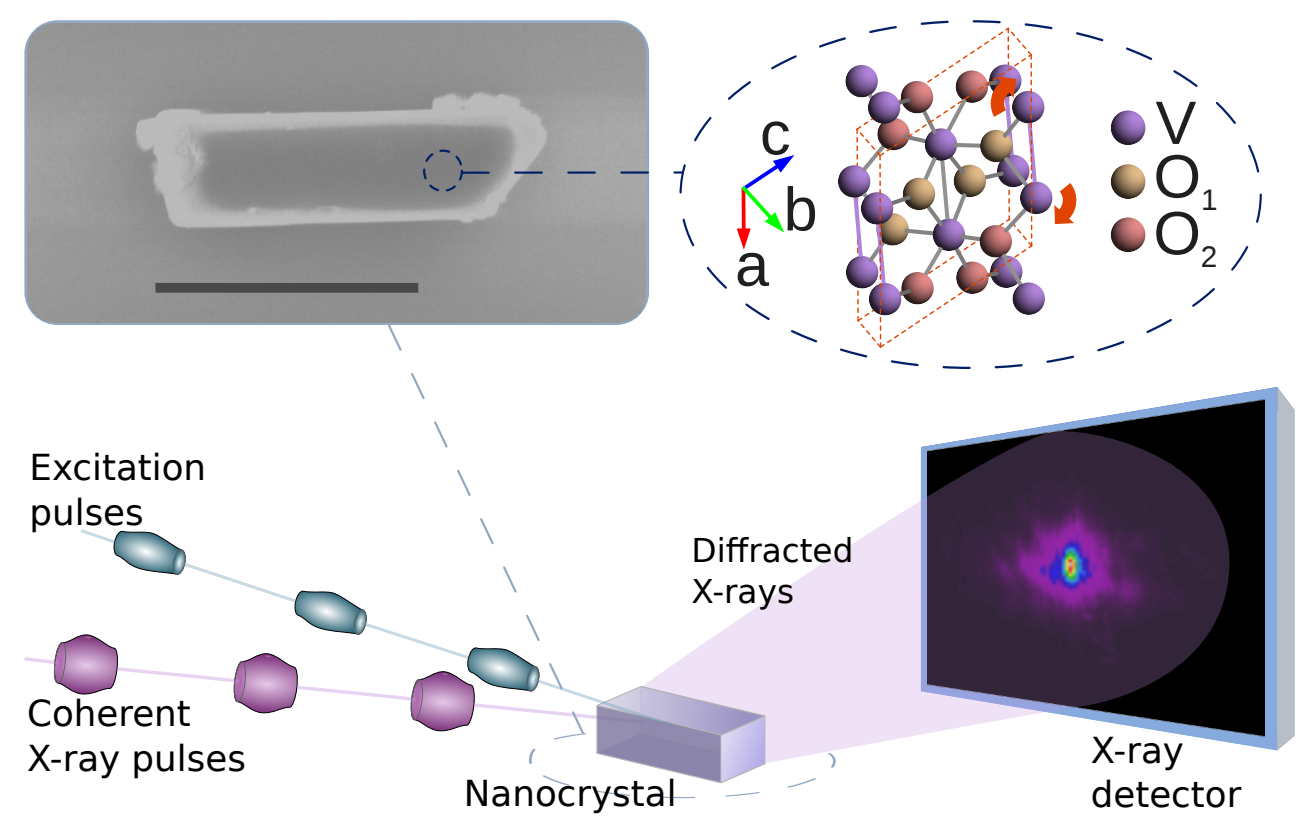

FIG. 1. Schematic of the experiment. The sample is mounted in the Bragg reflection geometry. At time $t$, the sample is excited with a femto-second pulse of light from the Ti:Sapphire laser. After a known time interval $\Delta t=t^{\prime}-t$, the sample is probed with a femto-second pulse from the XFEL. By varying $\Delta t$, we were able to obtain coherent diffraction information on the time evolution of the process. The inset shows a scanning electron micrograph image of a single vanadium dioxide $\left(\mathrm{VO}_{2}\right)$ nanocrystal on the surface of a $\mathrm{Si}(100)$ substrate. A 1 micron scale bar is shown. Also shown inset is an illustration of the atomic arrangement in the $\mathrm{VO}_{2}$ unit cell for the monoclinic $\left(\mathrm{M}_{1}\right)$ structure.

${ }_{80}$ The diffracted intensity is measured using an area 109 pulse of light from the Ti:Sapphire laser. After a 81 detector which is positioned far enough away from 110 known time interval $\Delta t=t^{\prime}-t$, the sample was probed 82 the sample to resolve the finest fringes of the coher- 111 with a femto-second pulse from the XFEL. By vary${ }_{83}$ ent diffraction pattern. Iterative phase reconstruction ${ }_{112}$ ing $\Delta t$, it was possible to obtain coherent diffraction ${ }_{84}$ methods are then used to recover the complex elec- ${ }_{113}$ information on the time evolution of the process. By ${ }_{85}$ tron density $\rho(\mathbf{r})$ and phase information $\phi(\mathbf{r})$. This 114 inverting the coherent diffraction pattern, a real-space ${ }_{86}$ in turn provides information on the atomic displace- 115 image of the object can be obtained. Further details ${ }_{87}$ ments throughout the volume of the crystals according 116 on data compilation can be found in the Supplemen${ }_{88}$ to the relation $\phi(\mathbf{r})=\mathbf{Q} \cdot \mathbf{u}(\mathbf{r})$, where $\mathbf{u}$ is the atomic ${ }_{117}$ tary Information.

89 displacement.[23]

$118 \quad$ Figure 1 illustrates the geometry of the experiment. 90 In the following we combine time resolved pump- ${ }_{119}$ The XFEL was operated with an unfocused beam ${ }_{91}$ probe measurements with coherent X-ray diffraction. 120 size of 250 microns in diameter. The photon energy ${ }_{92}$ This method is used to study structural changes in 121 and duration was $8.682 \mathrm{keV}$ and $\sim 10$ femto-seconds 93 self-assembled vanadium dioxide nanocrystals. Time- 122 respectively with a typical flux of $10^{9}$ photons per ${ }_{94}$ resolved measurements were achieved using a pump- ${ }_{123}$ pulse, after passing through a $\mathrm{Si}(111)$ double-crystal ${ }_{95}$ probe scheme consisting of a femto-second Ti:Sapphire ${ }_{124}$ monochromator.[25] The Ti:Sapphire excitation laser ${ }_{96}$ laser system as an optical pump and the XFEL as a ${ }_{125}$ was operated at a wavelength of 800 nanometers. ${ }_{97}$ femto-second probe. The XFEL is particularly suited ${ }_{126} \mathrm{~A}$ pulse energy of 300 micro Joules per pulse was 98 to the study of structural phase transitions in solid 127 achieved using a chirped pulse amplifier system. The 99 state materials due to its femto-second timing res- ${ }_{128}$ full-width-half-maximum of the excitation beam was 100 olution and spatial coherence. Measurements were ${ }_{129}$ reduced to 460 microns in size before impinging on the 101 performed at the SPring-8 Angstrom Compact Free ${ }_{130}$ sample. Both beams were caused to coincide at the 102 Electron Laser (SACLA) facility in Japan. SACLA ${ }_{131}$ sample surface with less than 100 microns accuracy. ${ }_{103}$ is a compact XFEL operating down to $\sim 0.6 \AA$ in ${ }_{132}$ Position jitter was not a major concern when using the ${ }_{104}$ wavelength. [24] Coherent diffraction experiments ${ }_{133}$ unfocussed XFEL beam for studying a single nanome${ }_{105}$ were performed in air on a single $\mathrm{VO}_{2}$ nanocrystal in ${ }_{134}$ tre scale crystal as the crystal was significantly smaller 106 the Bragg reflection geometry. Pump-probe measure- ${ }_{135}$ than the beam and fully illuminated. Timing between 107 ments were performed in the usual way. Namely, at 136 the pump and probe lasers was controlled using an all 108 time $t$, the sample was excited with a femto-second ${ }_{137}$ optical system with the initial delay time tuned using 

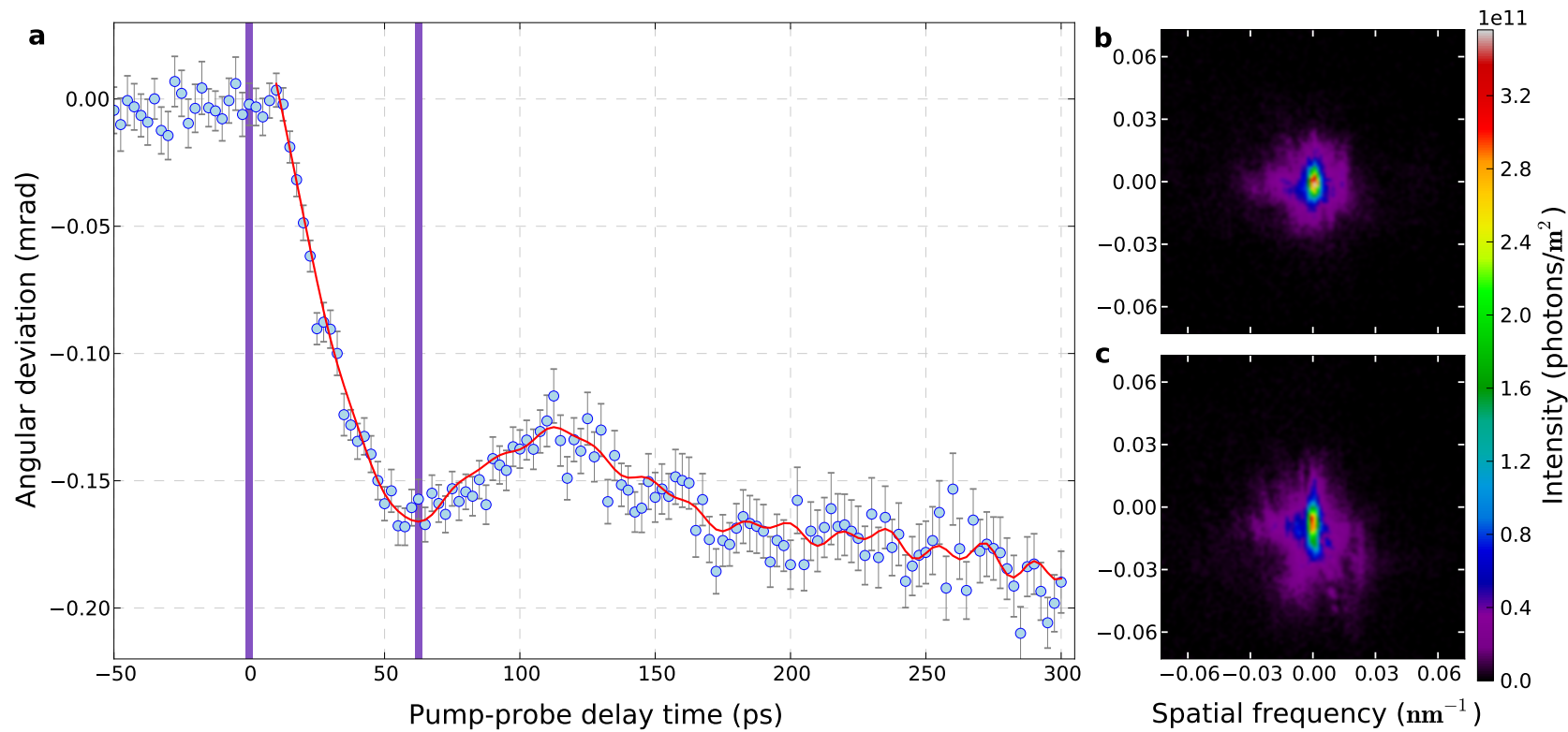

FIG. 2. Time dependent angular deviation of the coherent diffraction pattern. a, Deviation of the mean displacement of the coherent diffraction pattern in response to excitation at a fluence of $102 \mathrm{~mJ} / \mathrm{cm}^{2}$ per pulse from the Ti:Sapphire femto-second laser. b, Coherent diffraction pattern at delay time of 0 ps. c, Coherent diffraction pattern at delay time of $62.5 \mathrm{ps}$. Each diffraction pattern consists of the accumulation of data from 25 XFEL shots.

138 a fluorescent reference sample. The point zero time 170 herent diffraction pattern for varying delay times, in ${ }_{139}$ delay was calibrated down to an accuracy of $\sim 10 \mathrm{ps}$. 171 response to excitation at a fluence of $102 \mathrm{~mJ} / \mathrm{cm}^{2}$ ${ }_{140}$ The detector, XFEL and Ti:Sapphire laser were oper- ${ }_{172}$ per pulse from the Ti:Sapphire femto-second laser. ${ }_{141}$ ated at $20 \mathrm{~Hz}, 10 \mathrm{~Hz}$ and $5 \mathrm{~Hz}$ respectively allowing ${ }_{173}$ Measurements were performed in the low temperature 142 interleaving of background data and unperturbed co- 174 monoclinic Bragg geometry. Each data point results 143 herent diffraction data acquisition.

175 from the accumulation of data from 25 XFEL shots ${ }_{144} \mathrm{VO}_{2}$ nanocrystals were prepared using a chemical 176 with dark-field subtraction, as described in the Sup${ }_{145}$ vapour deposition process. An isolated single $\mathrm{VO}_{2} 177$ plementary Information. The delay interval between 146 nanocrystal was then prepared so that the (011) re- 178 each data point was 2.5 pico-seconds. Immediately af147 flection in the low temperature monoclinic phase is 179 ter time zero, a fast reduction in the Bragg angle was 148 approximately specular (see Methods section). In the 180 observed and occurred as the nanocrystal began to 149 high temperature tetragonal phase, the (110) reflec- 181 transition away from the monoclinic structure. This 150 tion is also approximately specular (see Supplemen- ${ }_{182}$ was followed by a slower reduction in the Bragg an151 tary Information). The sample was mounted on a ${ }_{183}$ gle. We understand this to result from the step-wise 152 custom built ceramic heated stage, designed by the ${ }_{184}$ motion of atoms in the unit cell during the transi153 authors, which was subsequently mounted onto a Ko- 185 tion. The rotation of the V-V pairs can be consid$154 \mathrm{hzu}(\phi, \chi, \theta)$ goniometer. The propagated wave was ${ }_{186}$ ered to occur in two stages. First a faster expansion 155 largely concealed in a vacuum path chamber with 187 of the vanadium pair and finally a slower shearing of 156 Kapton polyimide windows at either end between the 188 the pair. This is in agreement with previous studies 157 sample and detector. A multiport CCD X-ray de- 189 performed using electron diffraction on bulk samples. 158 tector was used to acquire coherent X-ray diffraction 190 [26] From the figure 2 we obtain a maximum speed of 159 patterns. The detector was mounted at a distance of 191 more than $0.007 \mathrm{~m} / \mathrm{s}$ for the expansion of the (011) ${ }_{160} 1410$ millimetres normal to the reflected wave and at 192 lattice planes. The (110) reflection of the high tem161 an angle of $2 \theta$, as illustrated in figure 1.

193 perature tetragonal structure is predicted to have a ${ }_{162}$ As the phase transition of $\mathrm{VO}_{2}$ exhibits a well de- ${ }_{194}$ Bragg angle of 0.99 mrad below that of the (011) re163 fined hysteresis between the thermodynamically sta- 195 flection of the monoclinic $\left(\mathrm{M}_{1}\right)$ structure and is there164 ble low temperature monoclinic and high temperature 196 fore ideal for observing both structures in tandem on 165 tetragonal phases, the pump-probe experiment was 197 a single detector plane. In order, however, to observe 166 performed below the hysteresis temperature. It was 198 femto-second angular deviations, it is essential that 167 found that $30^{\circ} \mathrm{C}$ was a sufficient temperature to avoid 199 diffraction is observed from a lattice plane with a sig168 hysteresis effects (see Methods section). $\quad 200$ nificant component in the plane of the V-V atom pair 169 Figure 2a shows the angular deviation of the co- 201 rotation. This is equivalent to Miller indices ( $h k l$ ) 

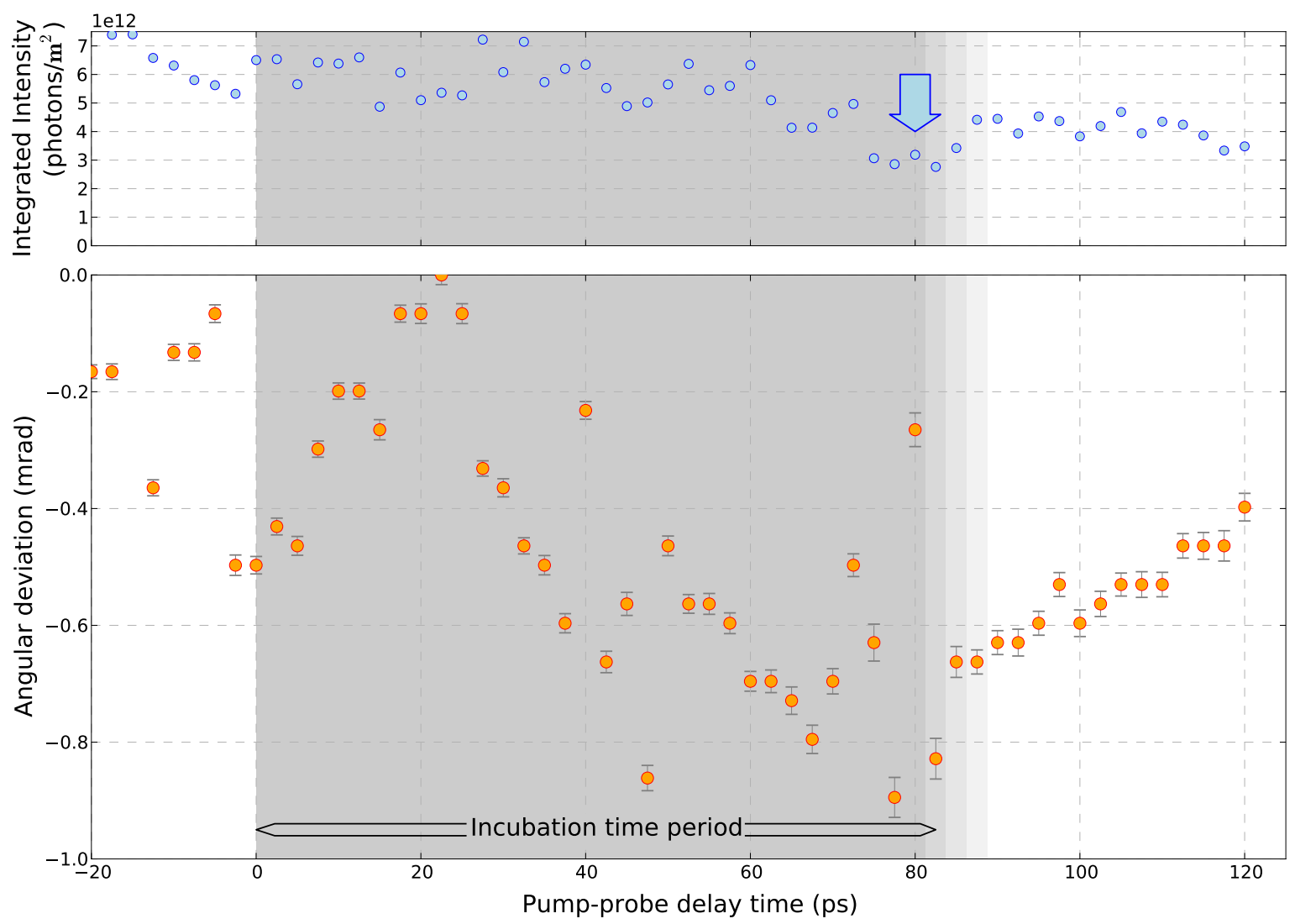

FIG. 3. Angular deviation and integrated intensity of the coherent diffraction pattern at increased excitation. Initial stages of the deviation of the displacement of the maximum intensity in response to excitation at a fluence of $306 \mathrm{~mJ} / \mathrm{cm}^{2}$ per pulse from the Ti:Sapphire femto-second laser excitation source. The integrated intensity is also shown and reduces by $\sim 4 \times 10^{12}$ photons $/ \mathrm{m}^{2}$ during the structural phase transition, as indicated. Each data point results from the accumulation of data from 25 XFEL shots with dark-field subtraction.

202 for which $h$ and $l$ are non-zero. Angular deviation of ${ }_{224}$ Each data point results from the accumulation of data 203 diffraction from the (011) reflection therefore occurs ${ }_{225}$ from 25 XFEL shots with dark-field subtraction. At 204 on the pico-second time scale as we have a significant 226 this excitation energy, we were able to observe a struc205 out of plane component of the transfer wavevector Q. ${ }_{227}$ tural transition. We found that during an incubation ${ }_{206}$ Oscillations in the angular deviation are also apparent ${ }_{228}$ period of $92.5 \mathrm{ps}$, the intensity peak of the coherent 207 and were best fitted to a damped harmonic oscillator 229 diffraction pattern moved between various locations ${ }_{208}$ equation with frequencies at $8.1 \mathrm{GHz}, 55.8 \mathrm{GHz}$ and ${ }_{230}$ within the diffraction pattern. This is likely due to in$20926.5 \mathrm{GHz}$ (see Supplementary Information). Coher- 231 ternal strain and plastic deformation of the nanocrys210 ent phonons propagating within the nanocrystal are ${ }_{232}$ tal. During the incubation period, the mean displace211 the likely cause where the oscillation frequencies are ${ }_{233}$ ment of the Bragg angle did not return to zero after 212 strongly coupled to the geometry of the nanocrystal. ${ }^{234}$ each excitation cycle suggesting that the crystal had ${ }_{213}$ Figures $2 \mathbf{b}$ and $2 \mathbf{c}$ show the coherent diffraction pat- 235 not completely returned to its original structure (see ${ }_{214}$ terns at delay times of $0 \mathrm{ps}$ and 62.5 ps respectively. ${ }_{236}$ supplementary information). This is likely due to the 215 Elongation of the coherent diffraction pattern occurs 237 crystal becoming trapped in an intermediate mono216 after 0 ps delay rather than a simple shift to lower an- ${ }_{238}$ clinic phase with a Bragg angle between that of the ${ }_{217}$ gle. This is a result of strain in the nanocrystal arising 239 monoclinic $\mathrm{M}_{1}$ and tetragonal phases. After an incu218 due to competing intermediate monoclinic $\left(\mathrm{M}_{2}\right)$ and 240 bation period of 92.5 ps the original coherent diffrac${ }_{219}$ triclinic phases as the lattice planes expand. $[8,27,28] 241$ tion pattern was no longer visible and a coherent new 220 Figure 3 shows the angular deviation of the coher- 242 diffraction pattern emerged. Toward the end of this ${ }_{221}$ ent diffraction pattern for varying delay times at an ${ }^{243}$ period and at a delay of $80 \mathrm{ps}$, the integrated intensity 222 increased excitation of $306 \mathrm{~mJ} / \mathrm{cm}^{2}$ per pulse from ${ }^{244}$ reduced by $\sim 4 \times 10^{12}$ photons $/ \mathrm{m}^{2}$ (as indicated in fig${ }_{223}$ the Ti:Sapphire femto-second laser excitation source. ${ }^{245}$ ure 3 ) for $12.5 \mathrm{ps}$, after which the coherent diffraction 

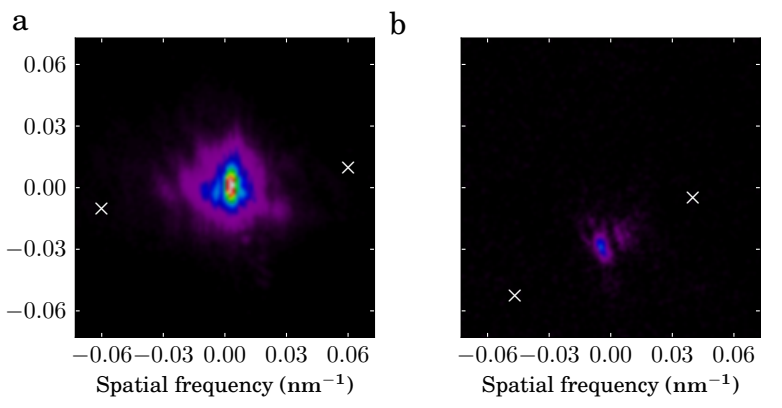

$\begin{array}{llllllllll}0.00 & 0.15 & 0.30 & 0.45 & 0.60 & 0.75 & 0.90 & 1.05 & 1.20 & 1.35\end{array}$

Intensity (photons $/ \mathrm{m}^{2}$ ) $\times 10^{11}$

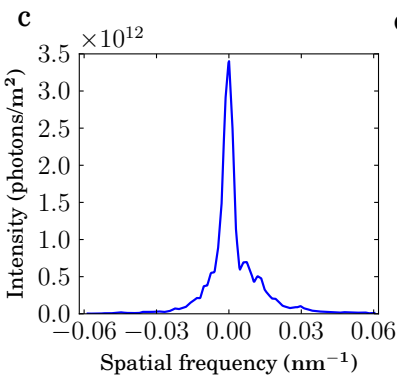

d

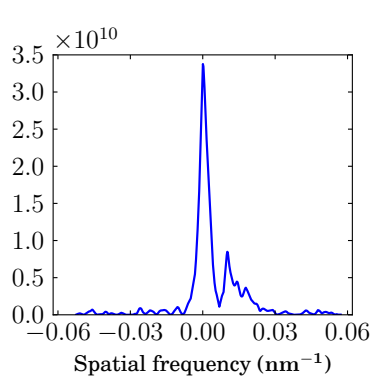

FIG. 4. Coherent diffraction pattern of monoclinic and tetragonal structures. Coherent diffraction patterns in response to excitation at a fluence of $306 \mathrm{~mJ} / \mathrm{cm}^{2}$ per pulse from the Ti:Sapphire femto-second laser at $\mathbf{a}, 0$ ps delay and b, $92.5 \mathrm{ps}$ delay. Intensity line scans through the points marked " $\times$ " are shown in $\mathbf{c}$ and $\mathbf{d}$, respectively.

${ }_{246}$ pattern of the new phase emerged.

247 Figure $4 \mathbf{a}$ and figure $4 \mathbf{b}$ shows the coherent diffrac${ }_{248}$ tion pattern correspondig to figure 3 at time delays of $2490 \mathrm{ps}$ and $92.5 \mathrm{ps}$ respectively. The displacement of the 250 centre of the Bragg peak of the new coherent diffrac251 tion pattern was at most 0.89 mrad below the original 252 agreeing reasonably well with theoretical predictions. ${ }_{253}$ We take into consideration that the diffractometer ${ }_{254}$ remained in the Bragg geometry for the monoclinic 255 phase during measurements and therefore only par256 tially satisfied that of the tetragonal phase. It is there${ }_{257}$ fore likely that the observed diffraction is from the ${ }^{258}$ high temperature tetragonal phase. Line scans drawn 259 between points marked " $\times$ " in figure $4 \mathbf{a}$ and figure $4 \mathbf{b}$ 260 are shown in figure $4 \mathbf{c}$ and $4 \mathbf{d}$, respectively. Compa${ }_{261}$ rable fringe spacings of 0.0046 and $0.0052 \mathrm{~nm}^{-1}$ are ${ }_{262}$ obtained for the monoclinic and tetragonal phase re263 spectively.

${ }_{264}$ In summary, we have performed time resolved co265 herent X-ray diffraction measurements on a single 266 nanoscale crystal of vanadium dioxide. We have ob${ }_{267}$ served ultra-fast dynamics of the phase transition 268 from monoclinic to tetragonal structure. Step-wise 269 motion of atoms in the unit cell was observed during ${ }_{270}$ the transition and attributed to rotation of the V-V 271 pairs. This study also demonstrates the feasibility of 272 the XFEL for the study of structural changes in corre- ${ }_{320}$ Promotion of Science (JSPS) Young Researcher Kak273 lated electronic materials. With further improvements 321 enhi award, grant number 24681014.
274 to iterative phase retrieval algorithms for reconstruct275 ing strained crystalline structures, it should also be 276 possible to invert coherent diffraction patterns from 277 time-resolved measurements to obtain real-space im278 ages. This ultimately will provide a means to obtain 279 real-space time-lapse images of the object with femto280 second resolution.

\section{METHODS}

282 Self-assembled $\mathrm{VO}_{2}$ nanocrystals were synthesised ${ }_{283}$ in bulk quantity using a high temperature chemical 284 vapour transport and deposition (CVTD) process at ${ }_{285} 900^{\circ} \mathrm{C}$ and at a pressure of 10 Pascals. A single $286 \mathrm{VO}_{2}$ nanocrystal was then transferred to a clean $\mathrm{Si}$ 287 (100) substrate, scored into four quadrants. Tem${ }_{288}$ perature dependent micro-Raman measurements were 289 then performed on a single $\mathrm{VO}_{2}$ nanocrystals to con290 firm the transition temperature. Subsequently, a sin${ }^{291}$ gle vanadium dioxide crystal was selected and pre292 pared on a Silicon substrate as shown in figure 1.

${ }_{293}$ The following procedure was used to determine 294 an appropriate initial sample temperature for pump295 probe measurements. The sample was heated to $30^{\circ} \mathrm{C}$. ${ }_{296} \mathrm{~A}$ coherent diffraction pattern from the low temper297 ature monoclinic (011) reflection was then recorded. ${ }_{298}$ The sample was subsequently heated through the 299 transition into the high temperature tetragonal phase 300 at which point the diffraction intensity disappeared. ${ }_{301}$ The temperature at which this occurred was recorded. ${ }_{302}$ The Bragg angle and sample orientation were then ${ }_{303}$ changed to locate the (110) reflection of the high tem${ }_{304}$ perature Rutile phase and a coherent diffraction pat305 tern was recorded. After this, the Bragg angle and 306 sample orientation was returned to that of the low 307 temperature (011) monoclinic reflection and the sam309 again visible. This process was found to be stable if 310 the sample was return to $30^{\circ} \mathrm{C}$. A temperature of $30^{\circ} \mathrm{C}$ 311 was therefore maintained throughout the experiment.

\section{ACKNOWLEDGEMENTS}

We would like to thank the operations staff scien314 tists at SACLA for the performance and flexibility of 315 the XFEL throughout our experiments. We would 316 also like to thank the software engineers for their ef${ }_{317}$ forts in producing a capable data acquisition system 318 with analysis capabilities.

This work was funded by the Japan Society for the 308 ple cooled until this coherent diffraction pattern was 
323 M.C.N., Y.N. and Y.T. planned the experiments. $324 \mathrm{VO}_{2}$ nanocrystals were prepared by M.C.N. Experi- ${ }^{384}$ 325 mental data was gathered by all authors. Data anal326 ysis was carried out by M.C.N. The manuscript was 327 prepared by M.C.N.

\section{* M.Newton@es.hokudai.ac.jp}

29 [1] Y. Tokura, Physics Today 56, 50 (2003).

[2] A. Tselev, J. D. Budai, E. Strelcov, J. Z. Tischler, 395 A. Kolmakov, and S. V. Kalinin, Nano Letters 11, 396 3065 (2011).

[3] M. Fiebig, K. Miyano, Y. Tomioka, and Y. Tokura, 398 Science 280, 1925 (1998).

[4] B. Kundys, M. Viret, D. Colson, and D. O. Kundys, 400 Nat Mater 9, 803 (2010). $\quad 401$

[5] G. Kotliar and D. Vollhardt, Physics Today 57, 53402 (2004).

[6] H. S. Park, O.-H. Kwon, J. S. Baskin, B. Barwick, 404 and A. H. Zewail, Nano Letters 9, 3954 (2009), pMID: 405 19856902.

[7] R. M. Wentzcovitch, W. W. Schulz, and P. B. Allen, 407 Phys. Rev. Lett. 72, 3389 (1994).

[8] A. Zylbersztejn and N. F. Mott, Phys. Rev. B 11, 409 4383 (1975).

[9] A. Cavalleri, T. Dekorsy, H. H. W. Chong, J. C. Kief- 411 fer, and R. W. Schoenlein, Phys. Rev. B 70, $161102{ }_{412}$ (2004).

[10] H.-T. Kim, Y. W. Lee, B.-J. Kim, B.-G. Chae, S. J. 414 Yun, K.-Y. Kang, K.-J. Han, K.-J. Yee, and Y.-S. 415 Lim, Phys. Rev. Lett. 97, 266401 (2006).

[11] G. Stefanovich, A. Pergament, and D. Stefanovich, 417 Journal of Physics: Condensed Matter 12, 8837418 (2000).

[12] Y. W. Lee, B.-J. Kim, J.-W. Lim, S. J. Yun, S. Choi, ${ }_{420}$ B.-G. Chae, G. Kim, and H.-T. Kim, Applied Physics Letters 92, 162903 (2008).

[13] T. Driscoll, H.-T. Kim, B.-G. Chae, M. D. Ventra, ${ }_{423}$ and D. N. Basov, Applied Physics Letters 95, 043503 (2009).

[14] C. Zhou, D. M. Newns, J. A. Misewich, and P. C. 42 Pattnaik, Applied Physics Letters 70, 598 (1997).

[15] G. Margaritondo and P. R. Ribic, Journal of Syn- ${ }^{428}$ chrotron Radiation 18, 101 (2011).

[16] M. M. Seibert, T. Ekeberg, F. R. N. C. Maia, 430 M. Svenda, J. Andreasson, O. Joensson, D. Odic, 431 B. Iwan, A. Rocker, D. Westphal, M. Hantke, D. P. 432 DePonte, A. Barty, J. Schulz, L. Gumprecht, N. Cop- 433 pola, A. Aquila, M. Liang, T. A. White, A. Mar- 434 tin, C. Caleman, S. Stern, C. Abergel, V. Seltzer, ${ }^{435}$ J.-M. Claverie, C. Bostedt, J. D. Bozek, S. Boutet, 436 A. A. Miahnahri, M. Messerschmidt, J. Krzywinski, ${ }^{437}$ G. Williams, K. O. Hodgson, M. J. Bogan, C. Y. 438 Hampton, R. G. Sierra, D. Starodub, I. Andersson, 439 S. Bajt, M. Barthelmess, J. C. H. Spence, P. Fromme, 440 U. Weierstall, R. Kirian, M. Hunter, R. B. Doak, 441 S. Marchesini, S. P. Hau-Riege, M. Frank, R. L. Shoe- 442 man, L. Lomb, S. W. Epp, R. Hartmann, D. Rolles, 443 A. Rudenko, C. Schmidt, L. Foucar, N. Kimmel, 444
P. Holl, B. Rudek, B. Erk, A. Hoemke, C. Reich, D. Pietschner, G. Weidenspointner, L. Strueder, G. Hauser, H. Gorke, J. Ullrich, I. Schlichting, S. Herrmann, G. Schaller, F. Schopper, H. Soltau, K.-U. Kuehnel, R. Andritschke, C.-D. Schroeter, F. Krasniqi, M. Bott, S. Schorb, D. Rupp, M. Adolph, T. Gorkhover, H. Hirsemann, G. Potdevin, H. Graafsma, B. Nilsson, H. N. Chapman, and J. Hajdu, Nature 470, 78 (2011).

[17] H. N. Chapman, P. Fromme, A. Barty, T. A. White, R. A. Kirian, A. Aquila, M. S. Hunter, J. Schulz, D. P. DePonte, U. Weierstall, R. B. Doak, F. R. N. C. Maia, A. V. Martin, I. Schlichting, L. Lomb, N. Coppola, R. L. Shoeman, S. W. Epp, R. Hartmann, D. Rolles, A. Rudenko, L. Foucar, N. Kimmel, G. Weidenspointner, P. Holl, M. Liang, M. Barthelmess, C. Caleman, S. Boutet, M. J. Bogan, J. Krzywinski, C. Bostedt, S. Bajt, L. Gumprecht, B. Rudek, B. Erk, C. Schmidt, A. Hoemke, C. Reich, D. Pietschner, L. Strueder, G. Hauser, H. Gorke, J. Ullrich, S. Herrmann, G. Schaller, F. Schopper, H. Soltau, K.U. Kuehnel, M. Messerschmidt, J. D. Bozek, S. P. Hau-Riege, M. Frank, C. Y. Hampton, R. G. Sierra, D. Starodub, G. J. Williams, J. Hajdu, N. Timneanu, M. M. Seibert, J. Andreasson, A. Rocker, O. Joensson, M. Svenda, S. Stern, K. Nass, R. Andritschke, C.-D. Schroeter, F. Krasniqi, M. Bott, K. E. Schmidt, X. Wang, I. Grotjohann, J. M. Holton, T. R. M. Barends, R. Neutze, S. Marchesini, R. Fromme, S. Schorb, D. Rupp, M. Adolph, T. Gorkhover, I. Andersson, H. Hirsemann, G. Potdevin, H. Graafsma, B. Nilsson, and J. C. H. Spence, Nature 470, 73 (2011).

[18] J. N. Clark, L. Beitra, G. Xiong, A. Higginbotham, D. M. Fritz, H. T. Lemke, D. Zhu, M. Chollet, G. J. Williams, M. Messerschmidt, B. Abbey, R. J. Harder, A. M. Korsunsky, J. S. Wark, and I. K. Robinson, Science 341, 56 (2013).

[19] J. Miao, P. Charalambous, J. Kirz, and D. Sayre, NATURE 400, 342 (1999).

[20] I. Robinson and J. Miao, MRS Bulletin 29, 177 (2004).

[21] M. Pfeifer, G. Williams, I. Vartanyants, R. Harder, and I. Robinson, Nature 442, 63 (2006).

[22] D. Sayre, Acta Crystallographica 5, 843 (1952).

425 [23] M. C. Newton, S. J. Leake, R. Harder, and I. K. Robinson, Nature Materials 9, 120 (2010).

[24] T. Ishikawa, H. Aoyagi, T. Asaka, Y. Asano, N. Azumi, T. Bizen, H. Ego, K. Fukami, T. Fukui, Y. Furukawa, S. Goto, H. Hanaki, T. Hara, T. Hasegawa, T. Hatsui, A. Higashiya, T. Hirono, N. Hosoda, M. Ishii, T. Inagaki, Y. Inubushi, T. Itoga, Y. Joti, M. Kago, T. Kameshima, H. Kimura, Y. Kirihara, A. Kiyomichi, T. Kobayashi, C. Kondo, T. Kudo, H. Maesaka, X. M. Marechal, T. Masuda, S. Matsubara, T. Matsumoto, T. Matsushita, S. Matsui, M. Nagasono, N. Nariyama, H. Ohashi, T. Ohata, T. Ohshima, S. Ono, Y. Otake, C. Saji, T. Sakurai, T. Sato, K. Sawada, T. Seike, K. Shirasawa, T. Sugimoto, S. Suzuki, S. Takahashi, H. Takebe, K. Takeshita, K. Tamasaku, H. Tanaka, R. Tanaka, T. Tanaka, T. Togashi, K. Togawa, A. Tokuhisa, H. Tomizawa, K. Tono, S. Wu, M. Yabashi, M. Yamaga, A. Yamashita, K. Yanagida, C. Zhang, T. Shintake, H. Kitamura, and N. Kumagai, Nat Photon 
445 advance online publication (2012), 10.1038/npho- 453 [27] T. M. Rice, H. Launois, and J. P. Pouget, Phys. Rev. ton.2012.141.

$454 \quad$ Lett. 73, $3042(1994)$

447 [25] Y. Inubushi, K. Tono, T. Togashi, T. Sato, T. Hat- 455 [28] A. Tselev, I. A. Luk'yanchuk, I. N. Ivanov, J. D. Busui, T. Kameshima, K. Togawa, T. Hara, T. Tanaka, 456 dai, J. Z. Tischler, E. Strelcov, A. Kolmakov, and H. Tanaka, T. Ishikawa, and M. Yabashi, Phys. Rev. 457 Lett. 109, 144801 (2012).

S. V. Kalinin, Nano Letters 10, 4409 (2010).

451 [26] P. Baum, D.-S. Yang, and A. H. Zewail, Science 318,

452 788 (2007). 